\title{
The intranasal endoscopic removal of schwannoma of the pterygopalatine and infratemporal fossae via the prelacrimal recess approach
}

\author{
Bing Zhou, MD,, Qian Huang, MD, ${ }^{1}$ Ping-Hung Shen, MD, ${ }^{2}$ Shun-Jiu Cui, MD, ${ }^{1}$ \\ Cheng-Shuo Wang, MD, ${ }^{1}$ Yun-Chuan Li, MD, ${ }^{1}$ Zhen-Kun Yu, MD, ${ }^{3}$ Xiao-Hong Chen, MD, ${ }^{1}$ and \\ Ting Ye, MD 4

\begin{abstract}
'Department of Otolaryngology-Head and Neck Surgery, Beijing Tongren Hospital, Capital Medical University, Key Laboratory of Otolaryngology-Head and Neck Surgery, Ministry of Education, Beijing; '2Department of Otolaryngology, Kuang-Tien General Hospital, Department of Biotechnology, Hung-Kuang University, Taichung, Taiwan; 32Department of Otolaryngology, Nanjing Tongren Hospital, Nanjing; and ${ }^{4}$ Department of Otolaryngology, Beijing Tiantan Hospital, Beijing, China
\end{abstract}

OBJECTIVE This study was undertaken to analyze the results of a novel surgical method-the endoscopic prelacrimal recess approach (PLRA) -in patients with tumors involving the pterygopalatine fossa (PPF) and infratemporal fossa (ITF). The surgical technique and indications for this approach are also discussed.

METHODS The authors analyzed data from 7 cases involving patients who underwent resection of PPF and ITF tumors by means of the endoscopic PLRA from 2004 to 2013. Preoperative and postoperative imaging studies were available in all cases and were reviewed. The surgical specimens were all confirmed to be schwannomas.

RESULTS All tumors were completely resected via endoscopic PLRA. There were no recurrences noted over a 28-month follow-up period. In 4 cases, the patients experienced postoperative facial numbness during the first two weeks after surgery, which gradually lessened thereafter. One patient continued to have mild facial numbness at most recent follow-up. The numbness had fully resolved in the other 3 cases.

CONCLUSIONS The intranasal endoscopic removal of schwannoma from PPF and ITF via PLRA can spare the whole lateral nasal wall, resulting in a reduction in morbidity. This is a novel minimally invasive surgical method for PPF and ITF tumors.

http://thejns.org/doi/abs/10.3171/2015.3.JNS132702

KEY WORDS prelacrimal recess; endoscopic sinus surgery; schwannoma; pterygopalatine fossa; infratemporal fossa; surgical technique

$\mathrm{T}$ UMORS originating or involving the pterygopalatine fossa (PPF) or infratemporal fossa (ITF) of the maxilla are uncommon, comprising only $0.5 \%$ of all head and neck tumors. ${ }^{2}$ Before the era of endoscopic surgery, lateral rhinotomy, midfacial degloving, the CaldwellLuc operation, and even a transmaxillary approach had been proposed for the resection of tumors of the PPF, but these approaches always resulted in some form of morbidity, such as facial scarring. After the introduction of endoscopic techniques, many papers were published describing an endonasal endoscopic approach and its application. . $^{1,68-11}$
The transpterygoid approach is commonly used when accessing the lateral recess of the sphenoid, the PPF, or the ITF, but this approach is often accompanied by cauterization of the sphenopalatine and vidian arteries and vidian nerve section. If the lesion involves the lateral aspect of the pterygoid process, the inferior turbinate has to be sacrificed; in addition, the frontal process of the maxilla limits surgical freedom. We developed a novel surgical method called the prelacrimal recess approach (PLRA), which can lead us directly to the PPF and ITF after creation and medialization of a "lacrimal sac and inferior turbinate flap."

ABBREVIATIONS IMA = internal maxillary artery; ITF = infratemporal fossa; PLRA = prelacrimal recess approach; PPF $=$ pterygopalatine fossa; TEM $=$ transnasal endoscopic maxillectomy.

SUBMITTED January 15, 2014. ACCEPTED March 12, 2015.

INCLUDE WHEN CITING Published online September 4, 2015; DOI: 10.3171/2015.3.JNS132702. 
We report here on 7 cases of PPF and ITF schwannomas resected by endoscopic PLRA and discuss the surgical procedure and its indications.

\section{Methods}

We collected data from 7 cases involving patients with PPF and IFT schwannomas who underwent endoscopic PLRA from 2004 to 2013. The group included 4 men and 3 women, and the patients' mean age was 43.6 years (range 29-59 years). Preoperative evaluation included CT and MRI studies and routine laboratory examinations. All patients underwent endoscopic PLRA under general anesthesia with controlled hypotension.

\section{Imaging Characteristics of the Lesions}

All of the lesions were located in the PPF and ITF but lateral to the sagittal plane of the lateral wall of nasal cavity. On CT, the lesions appeared as well-demarcated round or oval areas of soft tissue density over the PPF and ITF, with compression of the posterolateral wall of maxillary sinus, and bony absorption can be seen (Fig. 1) On MRI, the tumors appeared as well-demarcated lesions with high signal intensity both in T1- and T2-weighted images; cystic-like changes can be seen after enhancement (Fig. 2).

\section{Instrumentation}

We used the Karl Storz HD Endoscopy-Image 1 Camera System, with a $0^{\circ}$ endoscope (Explorent), and suctionbipolar forceps and other power instrumentation made by Medtronic.

\section{Surgical Procedure}

A detailed schematic illustration of this approach was presented in our previous publication (in a Chinese-language journal) on the use of the approach for patients with

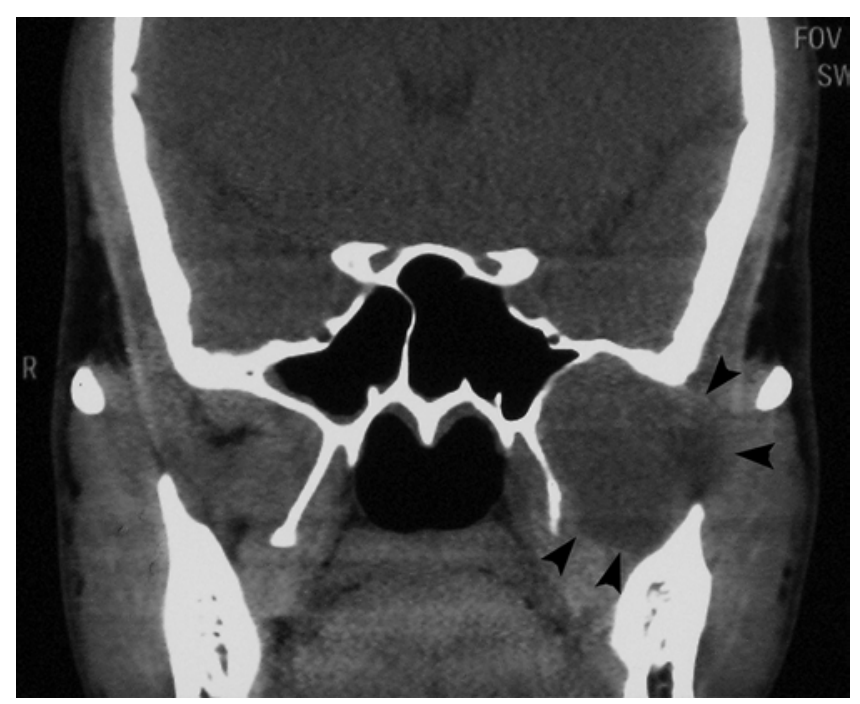

FIG. 1. Case 7. Preoperative CT scan demonstrating left PPF tumor (coronal reconstruction, soft tissue window). The tumor appears as a low-density, homogenous, well-circumscribed oval lesion in the PPF and ITF (arrowheads). Mild deformity and thinning of the left sphenoid sinus wall are also evident.

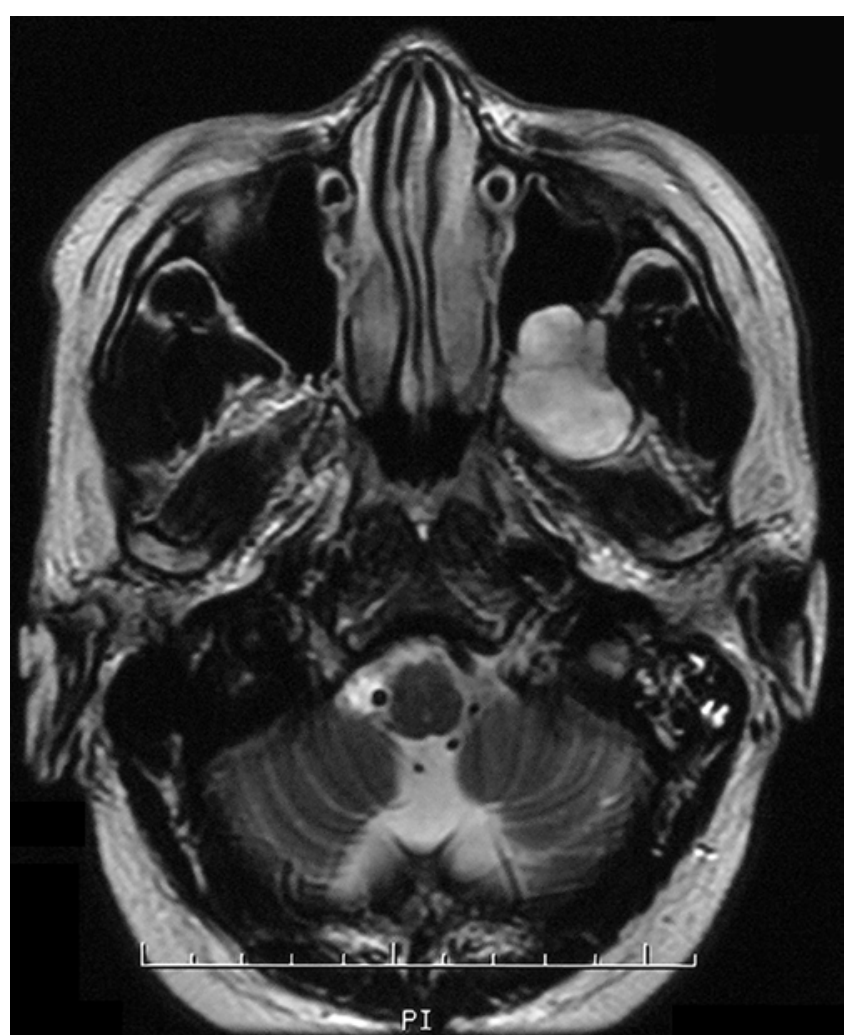

FIG. 2. Case 6. Preoperative axial T2-weighted MR image showing a high signal-density mass in the left PPF and ITF and remodeling of the adjacent posterior antral wall.

different lesions. ${ }^{11}$ A vertical incision is made through the cephalic end of the inferior turbinate. After elevation of the mucosal flap, a chisel and hammer are used to remove part of the frontal process of the maxilla, exposing the prelacrimal recess. Dissection then proceeds posteriorly, with particular care taken to avoid injury to the lateral wall of the lacrimal sac. Before entering the maxillary sinus, the lacrimal sac and inferior turbinate flap is medialized to gain exposure of the entire maxillary sinus. The sinus is entered, and the mucosa of the posterolateral wall is elevated and preserved, while the bony underlying part is removed. Part of the lateral pterygoid plate is also removed when necessary. The soft tissue of the PPF and ITF is then exposed. The internal maxillary artery (IMA) and its branches are carefully dissected. If the vasculature blocks access to the tumor, the IMA is either cauterized or clamped; the vasculature can be freed by either pushing it up or down, away from the tumor. During dissection, particular care is taken to avoid injury to the maxillary nerve and its terminal branch, the infraorbital nerve. After blunt dissection of the multilayered tumor capsules, we debulk the tumor and then remove it along with its capsule through the prelacrimal recess. If the tumor extends to the cavernous sinus, it can still be removed in the same fashion. The tumor cavity is then packed with NasoPore (Stryker) bioresorbable dressing, and the maxillary mucosa is repositioned and compressed with a water balloon. We perform an inferior antrostomy and place a water balloon catheter stent. Merocel (Medtronic) polyvinyl alcohol 


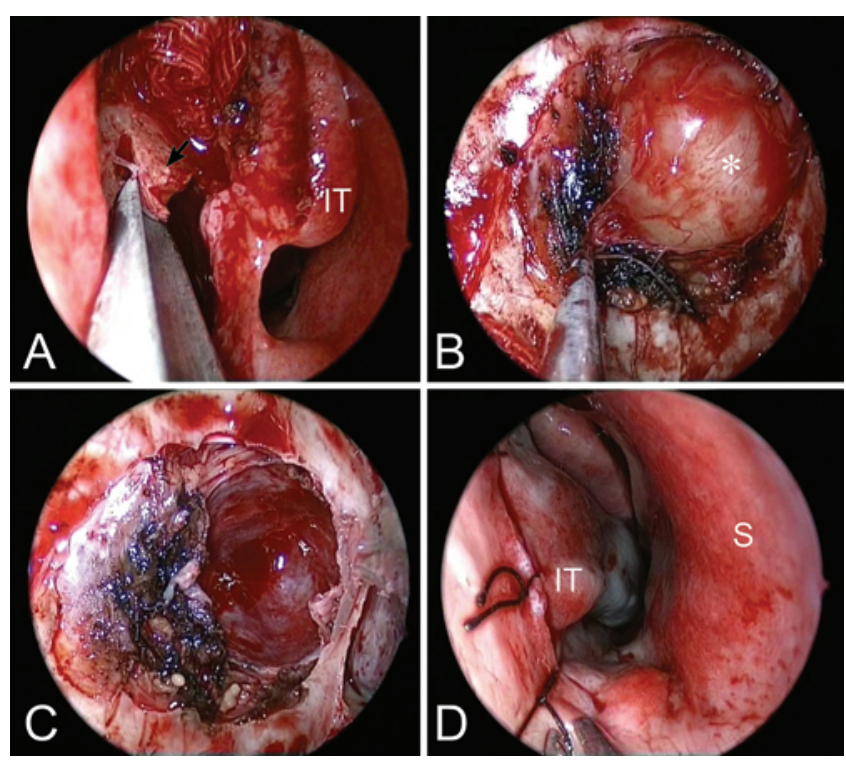

FIG. 3. Case 4. Intraoperative endoscopic images. The incision is made vertically through the cephalic end of the inferior turbinate $(\mathrm{A})$. We use the bony attachment of the inferior turbinate (arrow) as a landmark to expose the prelacrimal recess. The lacrimal sac and inferior turbinate flap is medialized before entering the maxillary sinus. The posterior bony wall of the maxillary sinus is removed, and the tumor (asterisk) is exposed layer by layer (B). After excision of the tumor (performed after core debulking in this case) (C), the flap is repositioned and sutured in place (D). IT = inferior turbinate; $S=$ septum. Figure is available in color online only.

sponge is then placed to compress the lateral wall of the nasal cavity (Fig. 3).

The water balloon is decompressed 1 day after surgery, and both the Merocel and water balloon are removed the following day. After removal of all nasal packing, an MRI examination is performed to provide a follow-up baseline. A CT scan is performed instead if the IMA was sacrificed with metal clamps.

\section{Results}

The demographic and clinical characteristics of the 7 patients are summarized in Table 1. The patients in Cases 1-3 presented with mild headaches, and those in Cases 4,5 , and 7 presented with facial, mouth angle, or dental numbness. The patient in Case 6 was found to have a tumor during the course of a routine physical examination.

All operations were performed under general anesthesia and with the use of an endoscope. There was minimal blood loss, and there were no complications. In Case 1, the tumor's consistency was hard and the IMA was blocking access, so the vasculature was sacrificed using clamps. In the remaining 6 cases, their tumors were soft, so the IMAs were preserved by pushing them out of the way. Total resection was achieved by means of tumor core debulking in Case 1-4 and 7, while in Cases 5 and 6 the tumor was resected in piecemeal fashion. In Case 1, the maxillary sinus was packed with iodine gauze due to substantial tumor bleeding; while, in the other cases the maxillary sinus was compressed with a water balloon and the lateral wall sutured, and an inferior antrostomy and water balloon catheter stent was placed.

All surgical specimens were confirmed to be schwannoma through postoperative histopathological examination (Fig. 4).

The mean operation time was 2.6 hours (range 1.5-5 hours), and the mean duration of hospital stay was 6.2 days (range 5-8 days). The mean follow-up period was 28 months (range 18-46 months). Four patients experienced postoperative facial numbness during the 1st and 2 nd weeks. In 3 of these cases, the numbness resolved fully; in the other case, the patient still had some mild facial numbness at the last follow-up evaluation (Case 3). The remaining 3 patients had no obvious morbidities (including dry eyes).

Postoperative endoscopy (usually performed 2 weeks after surgery and once every 3 months thereafter) revealed a well-healed incision wound and nasal mucosa with no crust formation; moreover, the inferior antrostomy was stabilized 1.5 months after surgery without stenosis. The maxillary mucosa edema subsided spontaneously within 3-6 months.

Postoperative imaging showed no deformity of the maxillary sinus, with soft tissue filling the lateral wall. As

TABLE 1. Summary of demographic and clinical characteristics of patients who underwent total resection of PPF and ITF schwannomas by means of an endoscopic PLRA

\begin{tabular}{|c|c|c|c|c|c|c|c|}
\hline $\begin{array}{l}\text { Case } \\
\text { No. }\end{array}$ & $\begin{array}{l}\text { Age } \\
\text { (yrs) }\end{array}$ & Sex & Symptoms & CT \& MRI Findings & Complications & $\begin{array}{l}\text { Follow-Up } \\
\text { (mos) }\end{array}$ & Outcome \\
\hline 1 & 42 & $\mathrm{~F}$ & Mild headache & $3 \times 4 \times 4 \mathrm{~cm}, \mathrm{rt}$ PPF \& ITF & No & 46 & Tumor free \\
\hline 2 & 53 & $\mathrm{~F}$ & Headache, mild diplopia & $\begin{array}{l}3 \times 3 \times 3 \mathrm{~cm} \text {, It PPF \& extension through inferior } \\
\text { orbital fissure to orbital apex }\end{array}$ & No & 39 & Tumor free \\
\hline 3 & 59 & M & Intermittent headache & $\begin{array}{l}3 \times 3 \times 3 \mathrm{~cm} \text {, It PPF \& ITF, extending to floor of } \\
\text { cavernous sinus }\end{array}$ & $\begin{array}{l}\text { Mild mouth angle } \\
\text { numbness }\end{array}$ & 34 & Tumor free \\
\hline 4 & 29 & M & $\begin{array}{l}\text { Occasional mouth angle } \\
\text { numbness }\end{array}$ & $\begin{array}{l}3 \times 3 \times 3 \mathrm{~cm}, \text { rt PPF \& ITF, extending to floor of } \\
\text { cavernous sinus }\end{array}$ & No & 23 & Tumor free \\
\hline 5 & 38 & $\mathrm{~F}$ & Facial protrusion \& numbness & $\begin{array}{l}6 \times 5 \times 4 \mathrm{~cm}, \text { rt PPF \& ITF partially invading } \\
\text { cavernous sinus }\end{array}$ & No & 19 & Tumor free \\
\hline 6 & 45 & M & None & $3 \times 3 \times 2.5 \mathrm{~cm}$, It PPF \& ITF & No & 18 & Tumor free \\
\hline 7 & 39 & M & Light facial numbness & $\begin{array}{l}4.5 \times 3 \times 3 \mathrm{~cm} \text {, It PPF \& ITF w/ partial invading to } \\
\text { cavernous sinus }\end{array}$ & No & 17 & Tumor free \\
\hline
\end{tabular}



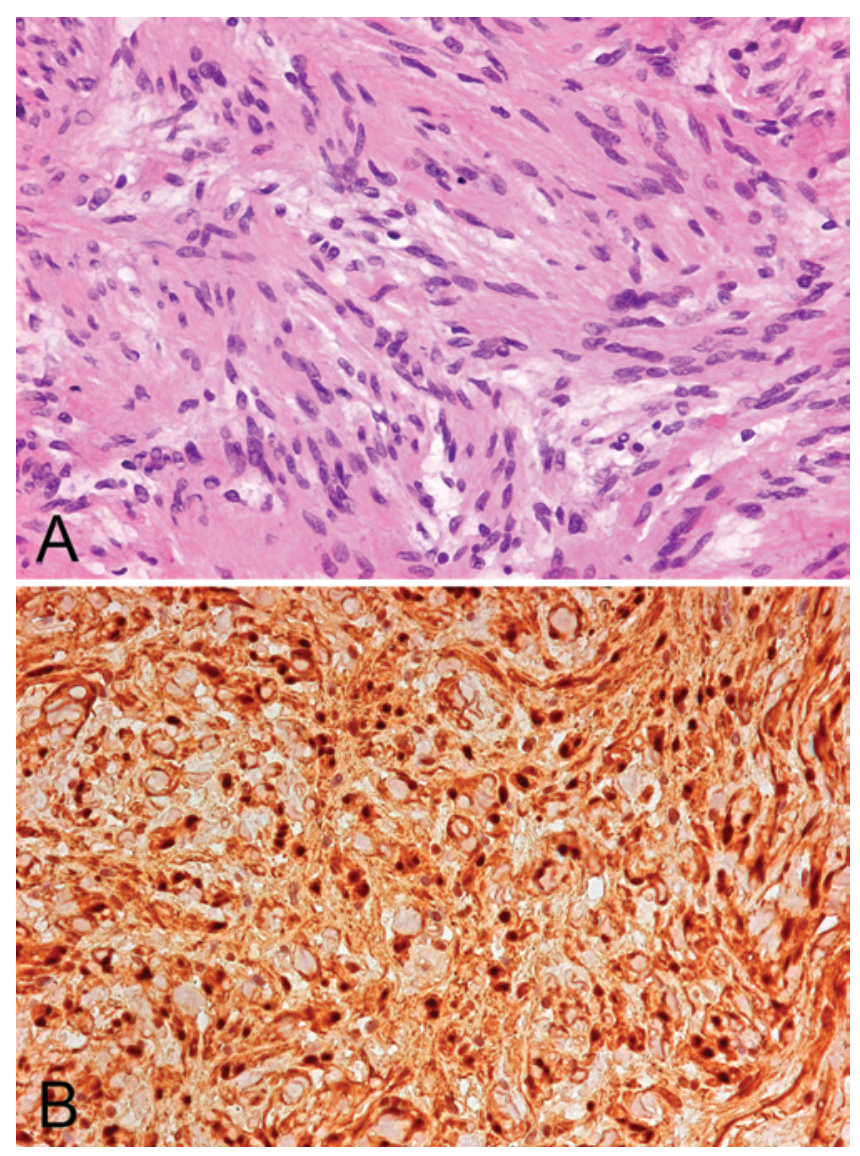

FIG. 4. Case 7. Photomicrographs showing characteristic histopathological features of schwannoma. A: An H \& E-stained section demonstrating a hypercellular Antoni $A$ area with fascicular arrangement of cells with fibrillar cytoplasm. The tumor cells are spindle shaped with oval nuclei. B: A tumor section immunostained for S-100 protein showing strong and diffuse reactivity in the cytoplasm and nuclei. Original magnification $\times 20$. Figure is available in color online only.

of the most recent follow-up evaluation, no patient showed any sign of tumor recurrence (Fig. 5).

\section{Discussion}

The PPF and ITF can be reached by an endoscopic endonasal approach. Anatomically, the anterior wall of the PPF and ITF is formed by the perpendicular plate of the palatine bone and the posterolateral wall of the maxillary sinus. These structures must be removed when performing an endoscopic endonasal approach to the PPF and ITF. Several approaches have been proposed to achieve wide exposure of the posterolateral wall of the maxillary sinus..$^{1,5,11}$ Transnasal endoscopic maxillectomy (TEM) is frequently used to accomplish this. Nicolai et al. described different types of resections in performing TEM (Types 1-3) according to their extent and location. Type 1 is a simple ethmoidectomy with middle antrostomy, sphenoidotomy, and frontal sinusotomy; Type 2 is a medial maxillectomy; and Type 3 involves removal of the medial third of the anterior wall of the maxillary sinus together with the nasolacrimal duct, providing a wide exposure of the entire maxillary sinus. ${ }^{5}$ An anatomical study by Alfieri et al. showed 3 differ- ent approaches to the PPF: the middle meatal transpalatine approach, the middle meatal transantral approach, and the inferior turbinectomy transantral approach. ${ }^{1}$ The first approach can expose the medial contents of the PPF; the second can expose the whole contents of PPF; and the third approach can give better access to ITF and provide a wider surgical field. These approaches can cause a wide array of morbidities, because the inferior turbinate, palatine bone, and sphenopalatine ganglion are frequently injured. If a PPF tumor is present without nasal cavity involvement, the middle meatal transpalatine approach still can be difficult, even if an angled endoscope is used. The septal window with contralateral nasal approach was developed to overcome this situation. ${ }^{3,7}$ Although the above approaches can provide a good surgical field, the inferior turbinate and lacrimal sac are frequently sacrificed; in addition, if a canine fossa approach is applied, it can sometimes cause postoperative cheek swelling and facial numbness. Our preliminary report on the PLRA describes a novel approach to the maxillary sinus when dealing with extensive maxillary sinus disease ${ }^{11}$ but we also found that the PLRA can be of benefit when approaching the PPF and ITF. The PLRA does not require excision of the perpendicular plate of the palatine bone; however, partial removal is sometimes necessary, but it does preserve the sphenopalatine ganglion and vidian nerve. Hofstetter et al. provided a step-by-step description of an approach to PPF and ITF on the basis of anatomy. ${ }^{4}$ Following a series of steps, a corridor to the ITF was established. For fully exposing the inferior aspect of the maxillary sinus, the inferior turbinate may need to be removed. ${ }^{3}$ The PLRA should preserve the inferior turbinate and nasal lacrimal duct. The incision is made vertically through the cephalic end of the inferior turbinate; the whole lacrimal sac is carefully preserved before exposing the prelacrimal recess of the maxillary sinus, along with medialization of the lacrimal sac and inferior turbinate flap, to expose the entire surgical view of the maxillary sinus. This entrance to the maxillary sinus looks narrow, but it is wide enough for lesions located in the maxillary sinus, PPF, or ITF. The cavity of the maxillary sinus provides a large space for manipulation, even with 3- or 4-hand techniques. The posterolateral wall of the maxillary sinus is also very easy to access through the PLRA without utilizing a bi-nostril transseptal technique. The anterior wall of the maxillary sinus was also carefully preserved to reduce injury to the alveolar nerves. All procedures were performed via a $0^{\circ}$ endoscope (Fig. 6). Before we finished the operation, the lacrimal sac and inferior turbinate flap were repositioned to their previous orientation (lateral wall), with the goal of this approach being to keep the nasal lateral wall intact. Our procedure can be categorized as a fourth approach that can be added to the 3 types of approaches proposed by Alfieri et al. ${ }^{1}$

After entering the PPF, preventing damage to the internal maxillary artery and nerve is crucial. The tumor pushes vessels and nerves out of the way, so the best method for avoiding injury to them is to dissect the tumor layer by layer. We used bipolar cauterization to cauterize fat tissue, then peeled the tumor capsule layer by layer until the final layer was encountered. We then carefully removed the tumor en bloc, along with this last layer of capsule. Minimal 

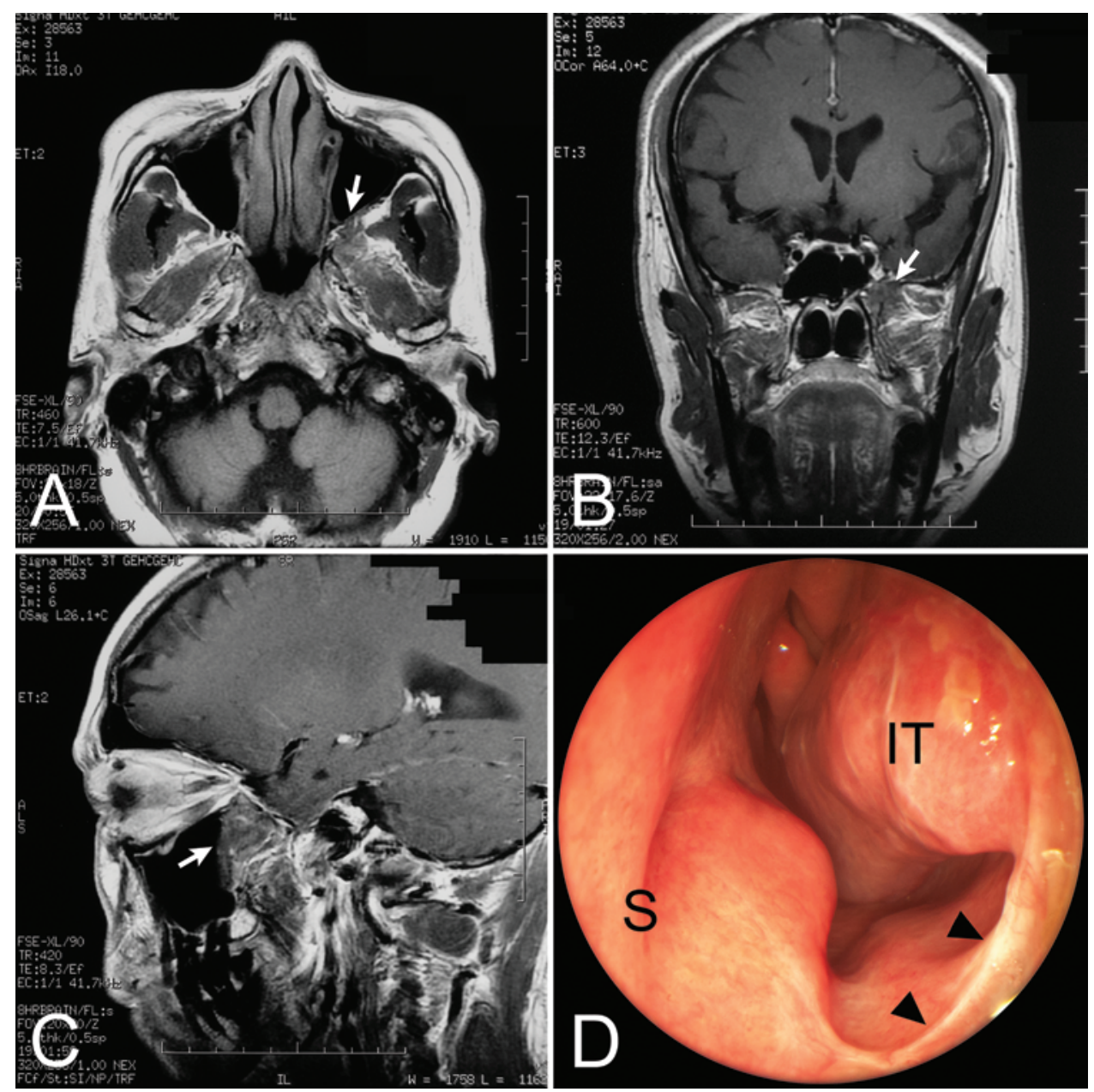

FIG. 5. Case 6. A-C: Postoperative T1-weighted contrast-enhanced MRI obtained 18 months after removal of a left PPF and ITF schwannoma (same patient as in Fig. 2) demonstrating the absence of tumor in the left PPF and ITF (white arrows) and normal maxillary sinus and PPF and ITF structures. D: Postoperative endoscopic view showing the integrated nasal cavity structures. The arrowheads indicate the healing incision. Figure is available in color online only.

bleeding can be achieved by this surgical method. If the tumor is particularly large, we can debulk the tumor through its core by means of a microdebrider. Making the tumor smaller helped us clearly identify the tumor margin and dissect it more easily. We encountered one tumor with a hard consistency and noted substantial bleeding when part of the capsule was peeled along with the tumor; as a result, iodine gauze was used to stop the bleeding. If the tumor spread out of the PPF and ITF into the nasal cavity, there might be some limitations using PLRA; in other words, the standard medial maxillectomy should be indicated in such cases. For the present series, however, because all of the tumors were located entirely within the PPF and ITF, we believe the PLRA was superior to the medial maxillectomy in terms of normal tissue preservation.

There were no intraoperative or postoperative CSF leaks in this case series. If a CSF leak does occur, free graft, such as fascia lata or temporal fascia, should be used for repair.

These 7 PPF and ITF schwannomas mainly originated from the second branch of the trigeminal nerve or the maxillary nerve or its branches. The tumors were found in
3 of the cases by investigating the cause of headaches, and in 3 other cases they were found during the investigation of facial, mouth angle, or dental numbness. In 1 case the tumor was found during the course of a routine physical examination. During tumor dissection and resection, the maxillary nerves were all carefully preserved. The main trunk of the maxillary nerve was protected even though there was unavoidable manipulation and minor branch injuries. Four patients experienced facial numbness in the maxillary nerve distribution for 1 to 2 weeks postoperatively. The area of numbness area gradually became restricted to the labrum over time, and in 3 of the 4 cases it resolved fully within 3 to 6 months after surgery. Only one of our patients had persistent numbness (mouth angle numbness) at long-term follow-up (Case 3). Three patients had no significant postoperative side effects. All patients were free of tumor during an average follow-up of 28 months (see Fig. 5). Furthermore, no sinusitis was noted during follow-up evaluations, so this may imply that the protection of the surrounding mucosa of maxillary sinus natural ostium may preserve its function of ventilation and drainage. 

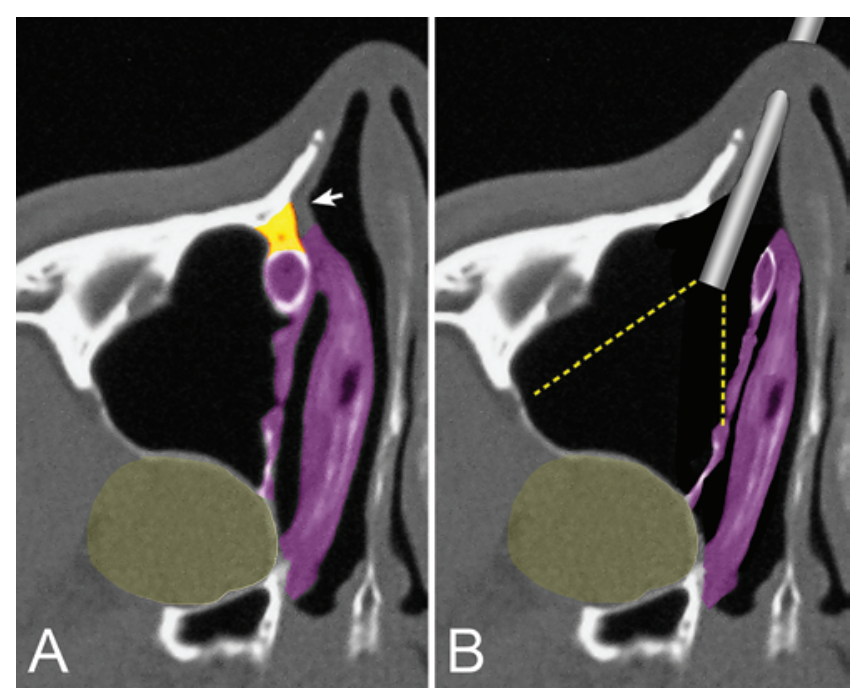

FIG. 6. Surgical drawings of PLRA. A: The incision is made vertically through cephalic end of inferior turbinate (arrowhead). A chisel and hammer are used to remove the bony structure of prelacrimal recess (yellow), and the lacrimal sac and inferior turbinate flap (purple) before entering the maxillary sinus. $\mathrm{B}: \mathrm{A} 0^{\circ}$ endoscope is introduced. The posterolateral wall of the maxillary sinus as well as the PPF and ITF can be manipulated very easily. Figure is available in color online only.

The advantages of the endoscopic PLRA are as follows. 1) This approach results in less injury, with neither cosmetic scarring nor neurovascular injury caused by a facial incision. 2) It provides an adequate operation field. To approach the maxillary sinus or the PPF and ITF via the posterolateral wall of the maxillary sinus, the PLRA may offer an adequate surgical field to allow a 4-handed operation and thus may reduce operation time. 3) It allows for preservation of sinus structure and function. After tumor resection, we may suture the incision wound in the nasal lateral wall to restore the structure. Postoperative care is easy, and wound healing is rapid.

\section{Conclusions}

Based on our experience with the 7 cases reported in this paper and our preliminary investigation, the indication for PLRA would be benign tumors located laterally to the lateral wall of the nose, especially with PPF and ITF involvement. In cases of schwannomas, due to their soft-tissue consistency with clear margins, we can apply the PLRA to reach the PPF or ITF for debulking surgery, even if the tumor has invaded the cavernous sinus or orbital apex. As for other tumors, especially malignant tumors, we have to gather more experience in anatomy and clinical technique with regard to the tumor biological characteristics to determine if the PLRA is feasible for malignant tumor removal.

\section{Acknowledgment}

This work was supported by a grant for training high-level health technicians of the Beijing health system to Bing Zhou (2009-3-36) and by the Health Ministry Special Fund (201202005).

\section{References}

1. Alfieri A, Jho HD, Schettino R, Tschabitscher M: Endoscopic endonasal approach to the pterygopalatine fossa: anatomic study. Neurosurgery 52:374-380, 2003

2. Bao S, Ni S, Zhang J, Li L, Mo D, Guo C, et al: Treatment of lesions involving both the infratemporal fossa and middle skull base. Surg Neurol 66 (Suppl 1):S10-S17, 2006

3. Harvey RJ, Sheehan PO, Debnath NI, Schlosser RJ: Transseptal approach for extended endoscopic resections of the maxilla and infratemporal fossa. Am J Rhinol Allergy 23:426-432, 2009

4. Hofstetter CP, Singh A, Anand VK, Kacker A, Schwartz TH: The endoscopic, endonasal, transmaxillary transpterygoid approach to the pterygopalatine fossa, infratemporal fossa, petrous apex, and the Meckel cave. J Neurosurg 113:967-974, 2010

5. Nicolai P, Tomenzoli D, Lombardi D, Maroldi R: Different endoscopic options in the treatment of inverted papilloma. Opera Tech Otolaryngol 17:80-86, 2006

6. Pasquini E, Sciarretta V, Farneti G, Ippolito A, Mazzatenta D, Frank G: Endoscopic endonasal approach for the treatment of benign schwannoma of the sinonasal tract and pterygopalatine fossa. Am J Rhinol 16:113-118, 2002

7. Ramakrishnan VR, Suh JD, Chiu AG, Palmer JN: Septal dislocation for endoscopic access of the anterolateral maxillary sinus and infratemporal fossa. Am J Rhinol Allergy 25:128-130, 2011

8. Robinson SR, Baird R, Le T, Wormald PJ: The incidence of complications after canine fossa puncture performed during endoscopic sinus surgery. Am J Rhinol 19:203-206, 2005

9. Wormald PJ, Ooi E, van Hasselt CA, Nair S: Endoscopic removal of sinonasal inverted papilloma including endoscopic medial maxillectomy. Laryngoscope 113:867-873, 2003

10. Wormald PJ, Robinson S: Transnasal endoscopic resection of benign tumors occurring from or extending into the infratemporal fossa. Opera Tech Otolaryngol 17:97-101, 2006

11. Zhou B, Han DM, Cui SJ, Huang Q, Wei YX, Liu HC, et al: [Endoscopic nasal lateral wall dissection approach to maxillary sinus.] Zhonghua Er Bi Yan Hou Tou Jing Wai Ke Za Zhi 42:743-748, 2007 (Chinese)

\section{Disclosure}

The authors report no conflict of interest concerning the materials or methods used in this study or the findings specified in this paper.

\section{Author Contributions}

Conception and design: Zhou. Acquisition of data: Zhou, Li, Yu, Chen, Ye. Analysis and interpretation of data: Huang, Cui, Wang. Drafting the article: Zhou, Shen. Critically revising the article: all authors. Reviewed submitted version of manuscript: all authors. Approved the final version of the manuscript on behalf of all authors: Zhou. Administrative/technical/material support: Zhou. Study supervision: Zhou. English translation: Shen.

\section{Correspondence}

Bing Zhou, Department of Otolaryngology-Head and Neck Surgery, Beijing Tongren Hospital, Capital Medical University, 1 Dongjiaominxiang, Dongcheng District, Beijing 100730, China. email: entzhou@263.net. 